\title{
New hyper-Kähler manifolds by fixing monopoles *
}

\author{
Conor J. Houghton ${ }^{\dagger}$ \\ DAMTP, Silver Street,Cambridge, CB3 9EW, United Kingdom
}

(February 1997)

\begin{abstract}
The construction of new hyper-Kähler manifolds by taking the infinite monopole mass limit of certain Bogomol'nyi-Prasad-Sommerfield monopole moduli spaces is considered. The one-parameter family of hyper-Kähler manifolds due to Dancer is shown to be an example of such manifolds. A new family of fixed monopole spaces is constructed. They are the moduli spaces of four $\mathrm{SU}_{4}$ monopoles, in the infinite mass limit of two of the monopoles. These manifolds are shown to be nonsingular when the fixed monopole positions are distinct.
\end{abstract}

\section{INTRODUCTION}

The moduli spaces of Bogomol'nyi-Prasad-Sommerfield (BPS) monopoles are hyper-Kähler manifolds. For charge two $\mathrm{SU}_{2}$ monopoles, the moduli space is the Atiyah-Hitchin manifold [1]. For two distinct monopoles in the maximally broken $\mathrm{SU}_{3}$ theory, the moduli space is Taub-NUT space [2, Since monopole moduli spaces have an isometric $\mathrm{SO}_{3}$ action corresponding to rotations of the monopoles in space, these hyper-Kähler manifolds are the only possible nontrivial four-dimensional monopole moduli spaces [5. [1]. In this paper other four-dimensional hyper-Kähler manifolds are derived from monopole moduli spaces by taking the infinite mass limit of some of the monopole masses, thus fixing the monopole positions. Fixing monopole positions generally breaks the $\mathrm{SO}_{3}$ isometry.

A one-parameter deformation of the Atiyah-Hitchin manifold is known, [6,7]. It was constructed using the hyperKähler quotient [B]. These hyper-Kähler manifolds are reinterpreted as BPS monopole moduli spaces, with one monopole fixed. A moduli space of BPS monopoles with two fixed monopoles is then considered. By constructing these moduli spaces via a hyper-Kähler quotient, they are proven to be nonsingular when the fixed monopoles are fixed at different points in space.

This paper is organized as follows. The Nahm formulation is reviewed in Sec. II. In Sec. III, Dancer's oneparameter family of hyper-Kähler manifolds is discussed. The moduli spaces with two fixed monopoles are introduced in Sec. IV and their nonsingularity is demonstrated in Sec. V. Other fixed monopole spaces are described in Sec. VI. The paper concludes in Sec. VII with some remarks about the applications of the new hyper-Kähler manifolds to three-dimensional supersymmetric theories. There is an Appendix concerning $\mathrm{SU}_{4}$ monopoles.

\section{NAHM DATA AND BPS MONOPOLES}

A BPS monopole is a pair $\left(\Phi, A_{i}\right)$ satisfying the Bogomol'nyi equation. The Higgs field $\Phi$ is an $\mathrm{su}_{n}$ valued scalar field and $A_{i}$ is the gauge potential. There is an $\mathrm{SU}_{n}$ gauge action on these fields, broken by the asymptotic Higgs field. If $\mathrm{SU}_{n}$ is broken to the maximal torus $\mathrm{U}_{1}^{n-1}$ the Higgs field at infinity is required to lie in the gauge orbit of

$$
\Phi_{\infty}=i \operatorname{diag}\left(t_{1}, t_{2}, \ldots, t_{n}\right) .
$$

By convention $t_{1}<t_{2}<\ldots<t_{n}$ and, since $\Phi$ is traceless, $t_{1}+t_{2}+\ldots+t_{n}=0$. Because of the asymptotic condition on $\Phi$, it gives a map from the large sphere at infinity into the quotient space

$$
\operatorname{orbit}_{\mathrm{SU}_{n}} \Phi_{\infty}=\mathrm{SU}_{n} / \mathrm{U}_{1}^{n-1} \text {. }
$$

Since $\pi_{2}\left(\mathrm{SU}_{n} / \mathrm{U}_{1}^{n-1}\right)=\mathbf{Z}^{n-1}$ the moduli space of monopoles is divided, topologically, into sectors labelled by $n-1$ integers, $k_{i}$, called topological charges. The maximal torus of $\mathrm{SU}_{n}$ is generated by the Cartan space and the matrix $\Phi_{\infty}$ defines a direction in this Cartan space. This direction picks out a unique set of simple roots in the Cartan space; those whose inner product with $\Phi_{\infty}$ is positive. Each $U_{1}$ in the maximal torus is generated by one of these simple

*Phys. Rev. D 56, 1120 (1997), hep-th/9702161

†Electronic address: C.J.Houghton@damtp.cam.ac.uk 
roots. The $k_{i}$ are then ordered by the requirement that adjacent $k_{i}$ 's correspond to non-orthogonal roots. A monopole with topological charge $\left(k_{1}, k_{2}, \ldots, k_{n-1}\right)$ is called a $\left(k_{1}, k_{2}, \ldots, k_{n-1}\right)$ monopole.

BPS monopoles can be constructed from Nahm data [9]. In the case of $\mathrm{SU}_{2}$ the space of $\mathrm{Nahm}$ data and the moduli space of monopoles are proven in [10] to be diffeomorphic and in [11] this diffeomorphism is proven to be isometric. The spaces are proven in [12] to be diffeomorphic when the unbroken symmetry group is Abelian. It is generally believed that the two spaces are isometric in all cases and in this paper this is assumed to be true.

The Nahm data corresponding to a $\left(k_{1}, k_{2}, \ldots, k_{n-1}\right)$ monopole are a triplet of skew-hermitian matrix functions defined over the interval $\left[t_{1}, t_{n}\right]$. The $t_{1}<t_{2}<\ldots<t_{n}$ subdivide the interval into $n-1$ abutting subintervals. For a $\left(k_{1}, k_{2}, \ldots, k_{n-1}\right)$ monopole a skyline diagram is drawn: a step function over the interval whose height on the $i$ 'th subinterval is $k_{i}$. For example, a $(3,1,2)$ monopole in an $\mathrm{SU}_{4}$ theory has diagram



The Nahm triplet is a triplet of square matrix functions of $t$ of different size over different subintervals. The size of the Nahm matrices in a subinterval is given by the height of the skyline in that interval. The matrices must satisfy the Nahm equations in each subinterval. The Nahm equations are

$$
\frac{d T_{1}}{d t}=\left[T_{2}, T_{3}\right]
$$

and two others by cyclic permutations of 1,2 and 3 .

There are boundary conditions relating the Nahm matrices in abutting subintervals. For the purpose of explaining these conditions lets us consider the skyline diagram

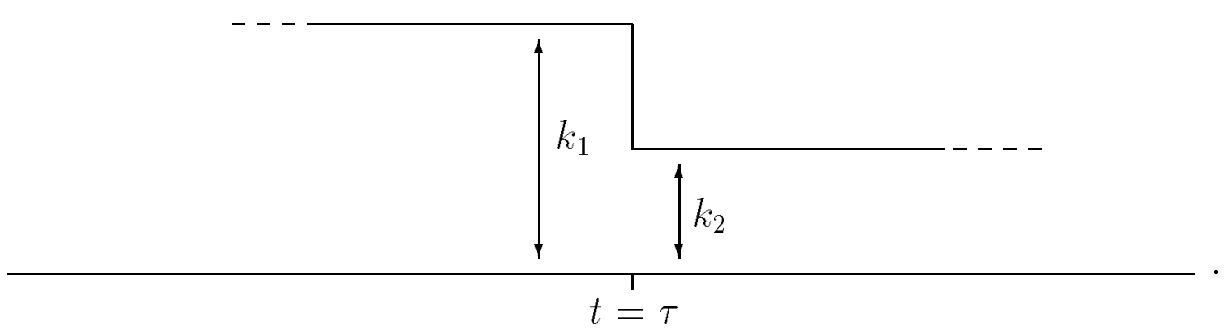

The skyline is $k_{1}$ high to the left of $\tau$ and $k_{2}<k_{1}$ high to the right of it. Thus, the Nahm triplet, $\left(T_{1}(t), T_{2}(t), T_{3}(t)\right)$, is a triplet of $k_{1} \times k_{1}$ matrices over the left interval and of $k_{2} \times k_{2}$ matrices over the right interval. As $t$ approaches $\tau$ from the left, it is required that

$$
T_{i}(s)=\left(\begin{array}{cc}
R_{i} / s+O(1) & O\left(s^{(m-1) / 2}\right) \\
O\left(s^{(m-1) / 2}\right) & T_{i}^{\prime}+O(s)
\end{array}\right)
$$

where $s=\tau-t, m=k_{1}-k_{2}$ and the $k_{2} \times k_{2}$ matrix $T_{i}^{\prime}$ is the nonsingular limit of the right interval Nahm data at $t=\tau$. The $m \times m$ residue matrices $R_{i}$ in (6) must form the irreducible $m$-dimensional representation of $\mathrm{su}_{2}$. Since the one-dimensional representation is trivial, there is no singularity when $m=1$. When $k_{1}$ is less than $k_{2}$, the conditions are almost the same, again there is a pole with residue matrices forming the $m=\left(k_{2}-k_{1}\right)$-dimensional representation of $\mathrm{su}_{2}$ and the $k_{1} \times k_{1}$ data are submatrices of the $k_{2} \times k_{2}$ data at the boundary. The situation when $k_{1}=k_{2}$ is very different; that case is not required in this paper.

When some of the $t_{i}$ 's in the asymptotic Higgs field are coincident, the residual gauge symmetry is enhanced. If two coincide, one $\mathrm{U}_{1}$ factor is replaced by an $\mathrm{SU}_{2}$ factor. If three coincide, two $\mathrm{U}_{1}$ 's are lost and an $\mathrm{SU}_{3}$ gained. Generally the unbroken group is $\mathrm{U}_{1}^{r} \times \mathrm{K}$ where $\mathrm{K}$ is a rank $n-r-1$ semisimple Lie group. Since $\pi_{2}\left(\mathrm{SU}_{n} /\left(\mathrm{U}_{1}^{k} \times \mathrm{K}\right)\right)=\mathbf{Z}^{r}$ monopole solutions in theories with non-Abelian residual symmetries have fewer topological charges. However, the monopole solutions still have $n-1$ integer labels. Some of these integers are the usual topological charges. The rest are what are known as holomorphic charges. 
The role of the holomorphic charges is subtle. If two $t_{i}$ 's are coincident, there is a zero thickness subinterval in the Nahm interval. The boundary conditions for Nahm data in this situation can be described in terms of those explained above, by formally imagining the zero thickness subinterval as the zero thickness limit of a subinterval of finite thickness. The Nahm data on this subinterval become irrelevant in the limit, but the height of the skyline on vanishing subintervals affects the matching condition between the Nahm matrices over the subintervals on either side.

An example is $\mathrm{SU}_{3}$ broken to $\mathrm{U}_{1}^{2}$. A $(2,1)$ monopole has skyline

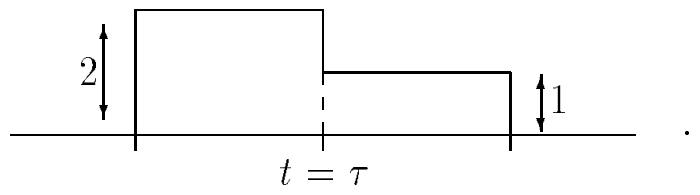

The Nahm data are $2 \times 2$ in the left interval and $1 \times 1$ in the right interval. The Nahm equations (4) dictate that $1 \times 1$ data are constant. Therefore, the right interval triplet is a triplet of imaginary numbers. These numbers are $i$ times the cartesian coordinates of the $(, 1)$ part of the $(2,1)$ monopole. The boundary conditions imply that the $2 \times 2$ data are nonsingular at the boundary, $t=\tau$, between the two intervals and, further, that their entries $T_{i}(\tau)_{2,2}$ are the $1 \times 1$ data. The $2 \times 2$ data are singular on the left boundary of the interval and the residues there form an irreducible representation of $\mathrm{su}_{2}$. Letting the right hand interval vanish, a $\mathrm{SU}_{3}$ monopole with topological charge two and holomorphic charge one is obtained. Holomorphic charges are distinguished from topological charges by square bracketing them. Thus, this monopole is a $(2,[1])$ monopole and it has skyline

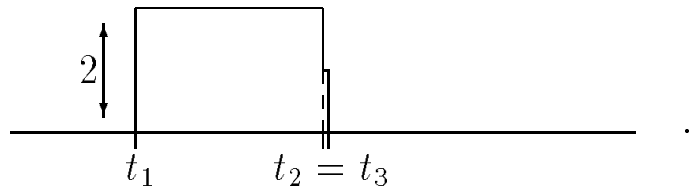

The Nahm data are $2 \times 2$ matrices with a pole on the left boundary but not on the right one.

In contrast, a $(2,0)$ monopole has skyline



The Nahm data are $2 \times 2$ matrices over the left hand subinterval and have poles at $t_{1}$ and $t_{2}$. There is no data over the right hand subinterval. These data are identical to $\mathrm{SU}_{2} 2$-monopole data and correspond to the embedding of an $\mathrm{SU}_{2}$ 2-monopole in $\mathrm{SU}_{3}$. The length of the right hand subinterval does not affect the Nahm data, there is a pole at both $t_{1}$ and $t_{2}$ irrespective of whether $t_{2}=t_{3}$ or not. If $t_{2}=t_{3}$ the Nahm data correspond to a $(2$, [0]) monopole.

These examples demonstrate how the holomorphic charges determine the boundary conditions and how these boundary conditions can be derived by imagining the non-Abelian case as the zero interval thickness limit of the Abelian case. It should be noted that different holomorphic charges do not necessarily correspond to different monopoles or to different Nahm data. For example, $(3,[1])$ monopoles can equally well be called $(3,[2])$ monopoles. This ambiguity is discussed, for example, by Weinberg in [13].

\section{DANCER'S FAMILY OF HYPER-KÄHLER MANIFOLDS}

In [6], the moduli space of centered $(2,[1])$ monopoles is constructed. These monopoles are charge $(2,[1]) \mathrm{SU}_{3}$ monopoles. They have the skyline diagram (8). They are called centered because their Nahm data are traceless. The moduli space, $M_{0}^{8}$, is eight dimensional. The Nahm data for such monopoles are a triplet of $2 \times 2$ traceless skew-hermitian matrix function over the interval $[-2,1]$. There is a simple pole at $t=-2$ and the residues there form the irreducible two-dimensional representation of $\mathrm{su}_{2}$. The space of such Nahm triplets, $M_{0}^{5}$, is five dimensional. The whole of $M_{0}^{8}$ is generated by the action of $\mathrm{SU}_{2}$ on these Nahm data. 
In the last section, for simplicity, the Nahm data described are gauge fixed. While the gauge fixed Nahm data are a triplet of matrix functions, to form the required $\mathrm{SU}_{2}$ orbit of $M_{0}^{5}$ the quadruplet of matrix functions $\left(T_{0}, T_{1}, T_{2}, T_{3}\right)$ is introduced. This quadruplet is required to satisfy the Nahm equations

$$
\frac{d T_{1}}{d t}+\left[T_{0}, T_{1}\right]=\left[T_{2}, T_{3}\right]
$$

and two others by cyclic permutations of 1,2 and 3 .

The introduction of $T_{0}$ allows a group action to be defined on the space of $(2,[1])$ Nahm data. If

$$
{ }_{0} \mathcal{G}=\left\{g \in C^{w}\left([-2,1], U_{2}\right): g(-2)=\mathbb{1}\right\}
$$

and its subgroup

$$
{ }_{0} \mathcal{G}_{0}=\left\{g \in C^{w}\left([-2,1], \mathrm{U}_{2}\right): g(-2)=g(1)=\mathbb{1}\right\}
$$

an action of $g \in{ }_{0} \mathcal{G}$ on $\left(T_{0}, T_{1}, T_{2}, T_{3}\right)$ is defined by

$$
\begin{aligned}
& T_{0} \mapsto g T_{0} g^{-1}-\frac{d g}{d t} g^{-1}, \\
& T_{i} \mapsto g T_{i} g^{-1}, \quad(i=1,2,3) .
\end{aligned}
$$

If $g \in{ }_{0} \mathcal{G}_{0}$ then the action is a gauge action. The moduli space of uncentered Nahm data, $M^{12}$, is the space of gauge inequivalent data. Furthermore, $\mathrm{U}_{2}={ }_{0} \mathcal{G} /{ }_{0} \mathcal{G}_{0}$ and, so, a $\mathrm{U}_{2}$ action on the data is given by Eq. (13). A hyper-Kähler quotient by the center of this $\mathrm{U}_{2}$ on $M^{12}$ centers the Nahm data, giving $M_{0}^{8}$. The remaining $\mathrm{SU}_{2}^{2}$ action can be fixed by setting $T_{0}$ to zero, reducing Eq. (10) to Eq. (4) and $M_{0}^{8}$ to $M_{0}^{5}$.

There is also an $\mathrm{SO}_{3}$ action. It both rotates the Nahm triplet as a vector and gauge transforms the four Nahm matrices. This action is not triholomorphic; it rotates the complex structures.

The $\mathrm{SU}_{2}$ action on $M_{0}^{8}$ is triholomorphic and isometric. This means that there is an induced moment map, $\mu$, from $M_{0}^{8}$ to $\mathbf{R}^{3}$ formed by the action of a $\mathrm{U}_{1}$ subgroup of $\mathrm{SU}_{2}$. Dancer's family of hyper-Kähler manifolds is the family of hyper-Kähler four manifolds

$$
M(\boldsymbol{\lambda})=\mu^{-1}(\boldsymbol{\lambda}) / \mathrm{U}_{1},
$$

where $\boldsymbol{\lambda} \in \mathbf{R}^{3}$. The $\mathrm{SO}_{3}$ action on $M_{0}^{8}$ is not an isometry of $M(\boldsymbol{\lambda})$, rather, it acts on $\boldsymbol{\lambda}$ to give an isometry between $M(\boldsymbol{\lambda})$ and $M(R \boldsymbol{\lambda})$ where $R$ is an $\mathrm{SO}_{3}$ matrix. $M(\mathbf{0})$ is a double cover of the Atiyah-Hitchin manifold.

The hyper-Kähler manifolds $M(\boldsymbol{\lambda})$ are hyper-Kähler quotients of a monopole moduli space. It is now shown that they are the infinite mass limit of another monopole space. The moment map $\mu$ is known explicitly. If the $\mathrm{U}_{1}$ subgroup is the subgroup which fixes $i \sigma_{3}$ when $\mathrm{SU}_{2}$ acts on $\mathrm{su}_{2}$ in the adjoint representation, the moment map $\mu: M_{0}^{8} \rightarrow \mathbf{R}^{3}$ given by this $\mathrm{U}_{1}$ action is

$$
\mu:\left(T_{0}, T_{1}, T_{2}, T_{3}\right) \mapsto\left(-\operatorname{trace}\left(T_{1}(1) i \sigma_{3}\right),-\operatorname{trace}\left(T_{2}(1) i \sigma_{3}\right),-\operatorname{trace}\left(T_{3}(1) i \sigma_{3}\right)\right) .
$$

The level set $\mu^{-1}(\boldsymbol{\lambda})$ consists of Nahm data whose entries $T_{i}(1)_{2,2}$ are $i \lambda_{i} / 2$ at $t=1$. For $(2,1)$ monopoles, (7), the data in the right-hand interval are given by the $T_{i}(1)_{2,2}$ entries of the left-hand Nahm data at the boundary. Thus, the hyper-Kähler manifolds $M(\boldsymbol{\lambda})$ are the moduli spaces of $(2,1)$ monopoles with the $(, 1)$ monopole fixed. The $(, 1)$ monopole can be fixed by taking its infinite mass limit. The monopole mass is proportional to the length of the corresponding interval, so this limit is



The vector $\boldsymbol{\lambda}$ is now related to the position of the $(, 1)$ monopole: the monopole whose position is fixed. When the position of the $(, 1)$ monopole is fixed in the center, the relative metric of the $(2$,$) monopole is Atiyah-Hitchin. That$ is not surprising. It has been noted, 14, 15, that if three $\mathrm{SU}_{2}$ monopoles are lined up, with suitable relative phases, the metric is Atiyah-Hitchin. 
An advantage of this description of $M(\boldsymbol{\lambda})$ is that its asymptotic behaviour may be calculated using the methods of [16 18], that is by approximating the monopoles by point particles and calculating their long range interactions. This yields a purely kinetic Lagrangian for the motion of the well separated monopoles and, hence, an asymptotic metric. This metric is

$$
d s^{2}=g_{i j} d \mathbf{x}_{i} \cdot d \mathbf{x}_{j}+g_{i j}^{-1}\left(d \chi_{i}+\mathbf{W}_{i k} \cdot d \mathbf{x}_{k}\right)\left(d \chi_{j}+\mathbf{W}_{j l} \cdot d \mathbf{x}_{l}\right)
$$

where, with no sum on repeated indices,

$$
\begin{aligned}
g_{j j} & =m_{j}-\sum_{i \neq j} \frac{\alpha_{i j}}{r_{i j}} \\
g_{i j} & =\frac{\alpha_{i j}}{r_{i j}}, \quad(i \neq j) \\
\mathbf{W}_{j j} & =-\sum_{i \neq j} \mathbf{w}_{i j}, \\
\mathbf{W}_{i j} & =\mathbf{w}_{i j}, \quad(i \neq j)
\end{aligned}
$$

and $\mathbf{x}_{i}, \chi_{i}$ and $m_{j}$ are the spacial coordinates, phases and masses of the monopoles; these are all well defined in the point particle approximation. A Dirac potential $\mathbf{w}(\mathbf{r})$ satisfies

$$
\nabla_{\mathbf{r}} \times \mathbf{w}=-\frac{\mathbf{r}}{r^{3}}
$$

In Eq. (18) $r_{i j}=\left|\mathbf{x}_{i}-\mathbf{x}_{j}\right|$ and $\mathbf{w}_{i j}$ is the corresponding Dirac potential. If the $i$ and $j$ monopoles have the same $\mathrm{U}_{1}$ charge then $\alpha_{i j}=1$ and if they correspond to adjacent $\mathrm{U}_{1}$ 's, $\alpha_{i j}=-1 / 2$, otherwise it is zero.

For $(2,1)$ monopoles

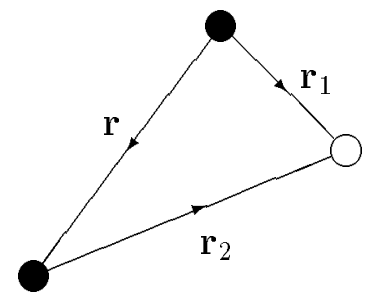

the mass of the two $(2$,$) monopoles is chosen to be one and that of the (, 1)$ monopole to be $m$. Then

$$
g_{i j}=\left(\begin{array}{ccc}
1-\frac{1}{r}+\frac{1}{2 r_{1}} & \frac{1}{r} & -\frac{1}{2 r_{1}} \\
\frac{1}{r} & 1-\frac{1}{r}+\frac{1}{2 r_{2}} & -\frac{1}{2 r_{2}} \\
-\frac{1}{2 r_{1}} & -\frac{1}{2 r_{2}} & m+\frac{1}{2 r_{1}}+\frac{1}{2 r_{2}}
\end{array}\right) .
$$

In the $\left(\mathbf{r}, \frac{1}{2}\left(\mathbf{x}_{1}+\mathbf{x}_{2}\right), \mathbf{x}_{3}-\frac{1}{2}\left(\mathbf{x}_{1}+\mathbf{x}_{2}\right)\right)$ basis this becomes

$$
g_{i j}^{\prime}=\left(\begin{array}{ccc}
\frac{1}{2}-\frac{1}{r}+\frac{1}{8 r_{1}}+\frac{1}{8 r_{2}} & 0 & \frac{1}{4 r_{2}}-\frac{1}{4 r_{1}} \\
0 & m+2 & m \\
\frac{1}{4 r_{2}}-\frac{1}{4 r_{1}} & m & m+\frac{1}{2 r_{1}}+\frac{1}{2 r_{2}}
\end{array}\right) .
$$

Thus, taking the infinite mass limit, the asymptotic metric on $M(\boldsymbol{\lambda})$ is

$$
\begin{aligned}
d s^{2} & =V_{1} d \mathbf{r} \cdot d \mathbf{r}+V_{2}^{-1}(d \chi+\mathbf{W} \cdot d \mathbf{r})^{2} \\
V_{1} & =\frac{1}{2}-\frac{1}{r}+\frac{1}{8 r_{1}}+\frac{1}{8 r_{2}} \\
V_{2} & =1-\frac{1}{r}+\frac{1-4 r_{1} r_{2}}{8 r_{1} r_{2}+2 r_{1}+2 r_{2}} \\
\mathbf{W} & =-\mathbf{w}+\frac{1}{8} \mathbf{w}_{1}+\frac{1}{8} \mathbf{w}_{2} .
\end{aligned}
$$

This metric is singular as $r \rightarrow 0$. It is only valid for large $r$.

The asymptotic metric for $M(\mathbf{0})$ is found by placing the fixed monopole at the center of mass of the two unfixed monopoles and, thus, by substituting $r_{1}=r_{2}=r / 2$ and $\mathbf{w}_{1}=\mathbf{w}_{2}=2 \mathbf{w}$ in Eq. (23). Making these substitutions reduces Eq. (23) to the asymptotic form of the Atiyah-Hitchin metric. 


\section{A NEW FAMILY OF HYPER-KÄHLER MANIFOLDS}

Another advantage of this description is that it immediately suggests a new family of four-dimensional hyperKähler manifolds, $N(\boldsymbol{\lambda}, \boldsymbol{\mu})$. In Sec. III, it is shown that $M(\boldsymbol{\lambda})$ is a fixed monopole space derived from the moduli space of charge $(2,1) \mathrm{SU}_{3}$ monopoles. This suggests that a new family of hyper-Kähler manifolds could be constructed by fixing monopoles in the moduli space of charge $(1,2,1) \mathrm{SU}_{4}$ monopoles. A $(1,2,1)$ monopole has skyline

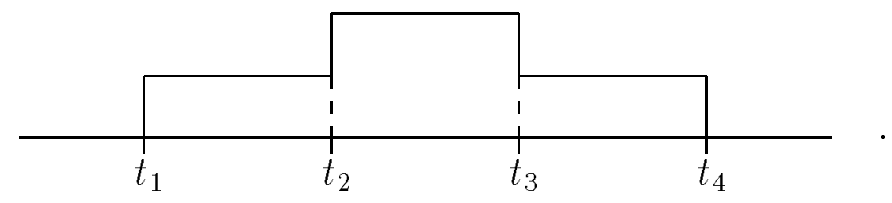

The corresponding Nahm data are $2 \times 2$ matrices in the middle subinterval and $1 \times 1$ matrices in the left and right subintervals. The Nahm data in the left subinterval are equal to the entries $T_{i}\left(t_{2}\right)_{2,2}$ of the $2 \times 2$ data, the Nahm data in the right subinterval are equal to the entries $T_{i}\left(t_{3}\right)_{2,2}$. All the Nahm data are analytic.

The limit where the subintervals $\left[t_{1}, t_{2}\right]$ and $\left[t_{3}, t_{4}\right]$ become infinitely long gives the $(1,2,1)$ fixed monopole spaces:



They are labelled by two vectors, $\boldsymbol{\lambda}$ and $\boldsymbol{\mu}$, the positions of the two fixed monopoles: the $(1,$,$) monopole and the$ $(,, 1)$ monopole. These spaces are denoted $N(\boldsymbol{\lambda}, \boldsymbol{\mu})$. The $\mathrm{SO}_{3}$ action on the charge $(1,2,1)$ moduli space is isometric and rotates the two vectors $\boldsymbol{\lambda}$ and $\boldsymbol{\mu}$. In the infinite mass limit of the $(1,$,$) monopole and the (,, 1)$ monopole, the action of some $R \in \mathrm{SO}_{3}$ gives an isomorphism between $N(\boldsymbol{\lambda}, \boldsymbol{\mu})$ and $N(R \boldsymbol{\lambda}, R \boldsymbol{\mu})$. Thus, $N(\boldsymbol{\lambda}, \boldsymbol{\mu})$ is a three-parameter family of hyper-Kähler manifolds. If $\boldsymbol{\lambda}$ and $\boldsymbol{\mu}$ are parallel then a $\mathrm{U}_{1}$ subgroup of the $\mathrm{SO}_{3}$ action fixes $N(\boldsymbol{\lambda}, \boldsymbol{\mu})$ and so $N(\boldsymbol{\lambda}, \boldsymbol{\mu})$ has a $\mathrm{U}_{1}$ isometry.

Using the same methods as in Sec. III, the asymptotic form of the $N(\boldsymbol{\lambda}, \boldsymbol{\mu})$ metric can be calculated. It is

$$
\begin{aligned}
d s^{2} & =V_{1} d \mathbf{r} \cdot d \mathbf{r}+V_{2}^{-1}(d \chi+\mathbf{W} \cdot d \mathbf{r})^{2} \\
V_{1} & =\frac{1}{2}-\frac{1}{r}+\frac{1}{8 r_{11}}+\frac{1}{8 r_{12}}+\frac{1}{8 r_{21}}+\frac{1}{8 r_{22}} \\
V_{2} & =1-\frac{1}{r}+\frac{1}{2} \frac{r_{11} r_{21}+r_{11} r_{22}+r_{12} r_{21}+r_{12} r_{22}-4 r_{11} r_{12} r_{21} r_{22}}{4 r_{11} r_{12} r_{21} r_{22}+r_{11} r_{12} r_{21}+r_{11} r_{12} r_{22}+r_{11} r_{21} r_{22}+r_{12} r_{21} r_{22}} \\
\mathbf{W} & =-\mathbf{w}+\frac{1}{8} \mathbf{w}_{11}+\frac{1}{8} \mathbf{w}_{12}+\frac{1}{8} \mathbf{w}_{21}+\frac{1}{8} \mathbf{w}_{22}
\end{aligned}
$$

where everything is defined as before, except that now there are two fixed monopoles and the distances from the two $(, 2$,$) monopoles to the first of these have been denoted by r_{11}$ and $r_{21}$ and the distances to the second by $r_{12}$ and $r_{22}$.

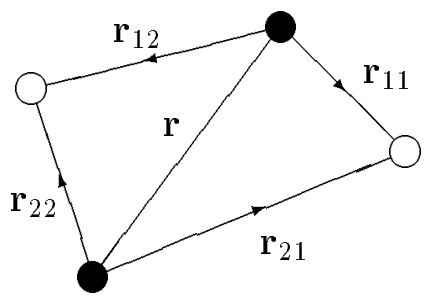

Examining the asymptotic formula, it is interesting to see how flat the $N(\boldsymbol{\lambda}, \boldsymbol{\mu})$ metrics are. All the metrics are flat up to the second order in $1 / r$. 


\section{NONSINGULARITY OF THE NEW HYPER-KÄHLER MANIFOLDS}

It is not clear from the discussion in Sec. IV that the $(1,2,1)$ moduli space remains nonsingular as the masses of the $(1,$,$) monopole and the (,, 1)$ monopole become infinite. Dancer's family; $M(\boldsymbol{\lambda})$, is known to be nonsingular because it can be constructed using a hyper-Kähler quotient. In imitation of this, $N(\boldsymbol{\lambda}, \boldsymbol{\mu})$ is constructed in this section by hyper-Kähler quotient of the moduli space of $([1], 2,[1])$ monopoles. These monopoles are $\mathrm{SU}_{4}$ monopoles of topological charge two in the theory where the residual symmetry is $\mathrm{SU}_{2} \times \mathrm{U}_{1} \times \mathrm{SU}_{2}$. The skyline diagram is



The Nahm data are $2 \times 2$ matrices analytic over the whole interval $[2,2]$. There are two commuting $\mathrm{SU}_{2}$ actions, one at each boundary. These data correspond to $([1], 2,[1])$ monopoles. In this section, the moduli space of ([1], 2, [1]) monopoles is used to construct $N(\boldsymbol{\lambda}, \boldsymbol{\mu})$ in the same way as the moduli space of $(2,[1])$ monopoles is used to construct $M(\boldsymbol{\lambda})$. It is found that the manifold $N(\boldsymbol{\lambda}, \boldsymbol{\mu})$ is free of singularities as long as $\boldsymbol{\lambda} \neq \boldsymbol{\mu}$.

The charge $([1], 2,[1])$ Nahm data are quadruplets $\left(T_{0}, T_{1}, T_{2}, T_{3}\right)$ satisfying the Nahm equations (10) and acted on by the gauge group

$$
{ }_{0} \mathcal{G}_{0}=\left\{g \in C^{w}\left([-2,2], \mathrm{U}_{2}\right): g(-2)=g(2)=\mathbb{1}\right\} .
$$

The two larger groups,

$$
\begin{aligned}
& { }_{0} \mathcal{G}=\left\{g \in C^{w}\left([-2,2], \mathrm{U}_{2}\right): g(-2)=\mathbb{1}\right\}, \\
& \mathcal{G}_{0}=\left\{g \in C^{w}\left([-2,2], \mathrm{U}_{2}\right): g(2)=\mathbb{1}\right\}
\end{aligned}
$$

are defined. These are subgroups of $\mathcal{G}=\left\{g \in C^{w}\left([-2,2], \mathrm{U}_{2}\right)\right\}$.

Two $\mathrm{U}_{2}$ actions are given by ${ }_{0} \mathcal{G} /{ }_{0} \mathcal{G}_{0}$ and $\mathcal{G}_{0} /{ }_{0} \mathcal{G}_{0}$. These actions commute. The whole $\mathrm{U}_{2} \times \mathrm{U}_{2}$ action is the $\mathcal{G} /{ }_{0} \mathcal{G}_{0}$ action. The center is $\mathrm{U}_{1} \times \mathrm{U}_{1}$. The Nahm data are fixed under the central element represented by the constant function $g(t)=e^{i \theta} \mathbb{1}_{2}$. The element represented by $g(t)=e^{i \theta t} \mathbb{1}_{2}$ sends $\left(T_{0}, T_{1}, T_{2}, T_{3}\right)$ to $\left(T_{0}-i \theta \mathbb{1}_{2}, T_{1}, T_{2}, T_{3}\right)$ and generates the vector field $\left(-i \mathbb{1}_{2}, 0,0,0\right)$. The hyper-Kähler quotient by this action centers the Nahm data. This space of centered data is called $N_{0}^{12}$. It is twelve dimensional. It has an isometric triholomorphic $\mathrm{SU}_{2} \times \mathrm{SU}_{2}$ action. There is also an $\mathrm{SO}_{3}$ action, which rotates $\left(T_{1}, T_{2}, T_{3}\right)$ as a three vector and commutes with the $\mathrm{SU}_{2} \times \mathrm{SU}_{2}$ action.

A $\mathrm{U}_{1} \times \mathrm{U}_{1}$ subgroup of the $\mathrm{SU}_{2} \times \mathrm{SU}_{2}$ is represented by the elements

$$
\alpha(t)=e^{\frac{i \theta}{4}(t+2) \sigma_{3}}, \quad \beta(t)=e^{\frac{i \theta}{4}(2-t) \sigma_{3}} .
$$

The moment map, $\mu: N_{0}^{12} \rightarrow \mathbf{R}^{3} \times \mathbf{R}^{3}$, for the action of this subgroup is

$$
\mu:\left(T_{0}, T_{1}, T_{2}, T_{3}\right) \mapsto(\boldsymbol{\lambda}, \boldsymbol{\mu})
$$

where

$$
\boldsymbol{\lambda}=\left(-\operatorname{trace}\left(T_{1}(-2) i \sigma_{3}\right),-\operatorname{trace}\left(T_{2}(-2) i \sigma_{3}\right),-\operatorname{trace}\left(T_{3}(-2) i \sigma_{3}\right)\right)
$$

and

$$
\boldsymbol{\mu}=\left(-\operatorname{trace}\left(T_{1}(2) i \sigma_{3}\right),-\operatorname{trace}\left(T_{2}(2) i \sigma_{3}\right),-\operatorname{trace}\left(T_{3}(2) i \sigma_{3}\right)\right) .
$$

By the same argument as in Sec. III, $N_{0}^{12}$ reduces to $N(\boldsymbol{\lambda}, \boldsymbol{\mu})$ under the hyper-Kähler quotient:

$$
N(\boldsymbol{\lambda}, \boldsymbol{\mu})=\mu^{-1}(\boldsymbol{\lambda}, \boldsymbol{\mu}) / \mathrm{U}_{1} \times \mathrm{U}_{1}
$$

The condition that $\boldsymbol{\lambda}$ and $\boldsymbol{\mu}$ must satisfy, in order for the $\mathrm{U}_{1} \times \mathrm{U}_{1}$ action to be free, are now needed. These are the conditions for the nonsingularity of the $N(\boldsymbol{\lambda}, \boldsymbol{\mu})$.

To apply these conditions it is necessary to solve the Nahm equations. Using the $\mathcal{G}$ action, $T_{0}$ is gauged to zero. This leaves an eight-dimensional space acted on by constant elements of $\mathcal{G}$ and by the $\mathrm{SO}_{3}$ action. By acting with 
the $\mathrm{SO}_{3}$ the $t$ invariants: $\operatorname{trace}\left(T_{1} T_{2}\right)$, trace $\left(T_{2} T_{3}\right)$ and trace $\left(T_{3} T_{1}\right)$, can be set to zero. This means that if the $T_{i}$ are written as

$$
T_{i}=\frac{1}{2} i f_{i} \mathbf{n}_{i} \cdot \boldsymbol{\sigma},
$$

where $i=1,2,3$ and the $\mathbf{n}_{i}$ are constant orthonormal vectors and so the functions $f_{1}, f_{2}$ and $f_{3}$ satisfy

$$
\frac{d f_{1}}{d t}=f_{2} f_{3}
$$

and two others given by cyclic permutations of 1,2 and 3 . The $\mathrm{SO}_{3}$ action are completely fixed by requiring that

$$
f_{1}^{2} \leq f_{2}^{2} \leq f_{3}^{2} .
$$

The remaining group action is that of constant elements of $\mathcal{G}$. It is fixed by setting $\mathbf{n}_{1}=(1,0,0), \mathbf{n}_{2}=(0,1,0)$ and $\mathbf{n}_{3}=(0,0,1)$. The resulting subspace of the moduli space $N_{0}^{12}$ is called $N^{3}$. Since the $\mathrm{SO}_{3}$ action on $N_{0}^{12}$ is not free, $N^{3}$ is not a manifold. Equations (38) are the well known Euler top equations and are solved in terms of Jacobi elliptic functions as

$$
\begin{aligned}
& f_{1}(t)= \pm \frac{D c n_{k}(D(t+\tau))}{s n_{k}(D(t+\tau))}, \\
& f_{2}(t)= \pm \frac{D d n_{k}(D(t+\tau))}{s n_{k}(D(t+\tau))} \\
& f_{3}(t)= \pm \frac{D}{s n_{k}(D(t+\tau))},
\end{aligned}
$$

where $0 \leq k \leq 1$ is the elliptic modulus, $D$ and $\tau$ are arbitrary real constants and the signs are all minus or exactly two of them are plus. Analyticity of the data requires that $\tau>2$ and $D(\tau+2)<2 K(k)$, where $4 K(k)$ is the period of $s n_{k}$. Further solutions are found by changing the sign of all three $f_{i}$ 's and sending $t$ to $-t$. The analyticity requirements on these further solutions are that $\tau<2$ and $D(\tau+2)<2 K(k)$. This exhausts all the solutions consistent with the various conditions which have been imposed.

The action of $\alpha(t)$ and $\beta(t)$ is given by Eq. (32). Since $T_{0}$ is zero on $N^{3}$ the only element in the group generated by $\alpha(t)$ and $\beta(t)$ which could have a fixed point in $N^{3}$ is the constant one $\alpha \beta(t)=e^{i \theta \sigma_{3}}$. For $\alpha \beta$ to have a fixed point in $N^{3}$ it is necessary and sufficient that $f_{1}(0)=f_{2}(0)=0$. This only occurs if $k=1$ and $\tau=\infty$. The solutions Eq. (40) are then $f_{1}(t)=0, f_{2}(t)=0$ and $f_{3}(t)=D$ and the hyper-Kähler quotient gives the space $N((0,0, D),(0,0, D))$. This means $N(\boldsymbol{\lambda}, \boldsymbol{\mu})$ with $\boldsymbol{\lambda}=\boldsymbol{\mu}=(0,0, D)$ may have a singularity. By considering the action on $N^{3}$ of $\mathrm{SU}_{2} \times \mathrm{SU}_{2}$, it is seen that the only points in $N_{0}^{12}$ where the action of $\alpha(t)$ or $\beta(t)$ is not free are those points in the $\mathrm{SO}_{3} \times\left(\right.$ constant elements of $\mathcal{G}$ ) orbit of the fixed points occuring in $N^{3}$. Therefore, the only potentially singular $N(\boldsymbol{\lambda}, \boldsymbol{\mu})$ manifolds are $N(\boldsymbol{\lambda}, \boldsymbol{\lambda})$. In the fixed monopole description, these are the manifolds of coincident fixed monopoles.

The manifold $N(\mathbf{0}, \mathbf{0})$ is singular. This is in contrast with $M(\mathbf{0})$ which is a double cover of the Atiyah-Hitchin manifold. The $N(\boldsymbol{\lambda}, \boldsymbol{\mu})$ spaces are not deformations of a smooth $\mathrm{SO}_{3}$ isometric hyper-Kähler manifold. It would be interesting to understand more of the geometry and topology of these spaces.

\section{OTHER FIXED MONOPOLE SPACES}

Following the example of $M(\boldsymbol{\lambda})$ and $N(\boldsymbol{\lambda}, \boldsymbol{\mu})$ it is natural to ask whether further nonsingular fixed monopole spaces might be constructed by fixing larger numbers of monopoles. For example, a large class of four-dimensional hyper-Kähler manifolds might be derived from the $\left(k_{1}, 2, k_{2}\right)$ monopole moduli spaces. One might conjecture that, as long as the $\left(k_{1},,\right)$ monopoles and the $\left(,, k_{2}\right)$ monopoles are not fixed in coincident positions, new multi-parameter families of four-dimensional hyper-Kähler manifolds could result.

More complicated mixtures of fixed and unfixed monopoles could be used to give fixed monopole spaces of dimensions higher than four. Fixed charges are distinguished from other charges by enclosing them in curly brackets. It could be conjectured that for $r>1$ the $\left(\left\{k_{1}\right\}, l_{1}, l_{2}, \ldots, l_{r},\left\{k_{2}\right\}\right)$ spaces are nonsingular when the $\left(k_{1}, \ldots,,\right)$ monopoles and the $\left(,, \ldots, k_{2}\right)$ monopoles are each fixed so they are not coincident with monopoles of the same type.

The asymptotic metrics can always be constructed for fixed monopole spaces using the point monopole methods of 116 18]. Generally, these asymptotic fixed monopole metrics are singular. This is not the case for the $(\{k\}, 1)$ space. 
In the limit of infinite $(k$,$) monopole mass the (k, 1)$ monopole asymptotic metric is the $k$ center multi-Taub-NUT metric of Hawking [19]. The positions of the $k$ centers are the $k$ fixed monopole positions. Since the multi-Taub-NUT metric is generically nonsingular and is the same asymptotically as the $(k, 1)$ metric, it seems likely that they are the same everywhere. Certainly, the $(1,1)$ monopole metric is known explicitly [2, 4 ] and the $(\{1\}, 1)$ metric is Taub-NUT. The $(1,1,1)$ metric is also known [18,20] and the infinite mass limit $(\{1\}, 1,\{1\})$ is two center multi-Taub-NUT.

Mixtures of fixed, topological and holomorphic charges might also be considered. An example is the eightdimensional space $(\{1\}, 2,[1])$ :

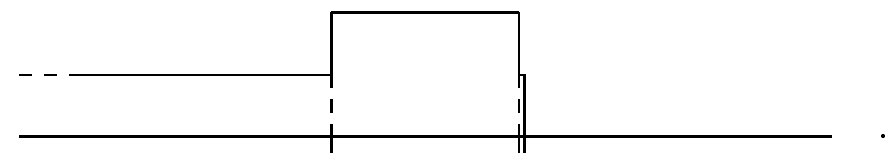

It is an interesting space, it has a tri-holomophic $\mathrm{SU}_{2}$ isometry and an isometric $\mathrm{U}_{1}$ action which rotates the complex structures.

\section{APPLICATIONS}

The $N(\boldsymbol{\lambda}, \boldsymbol{\mu})$ are gravitational instantons. Gravitational instantons are asymptotically flat solutions of the vacuum Einstein equations. All asymptotically flat four-dimensional hyper-Kähler manifolds are gravitational instantons. As noted earlier, $N(\boldsymbol{\lambda}, \boldsymbol{\mu})$ approaches flat space very rapidly.

Fixed monopole spaces are relevant to $(2+1)$-dimensional quantum field theories. In a celebrated recent paper, [21, Hanany and Witten propose a correspondence between three-dimensional supersymmetric gauge theories and moduli spaces of magnetic monopoles. In the language of [21] the fixed monopole spaces correspond to brane configurations in which some of the threebranes are infinitely extended in the direction along which the fivebranes are separated. Thus, $N(\boldsymbol{\lambda}, \boldsymbol{\mu})$ corresponds to the configuration

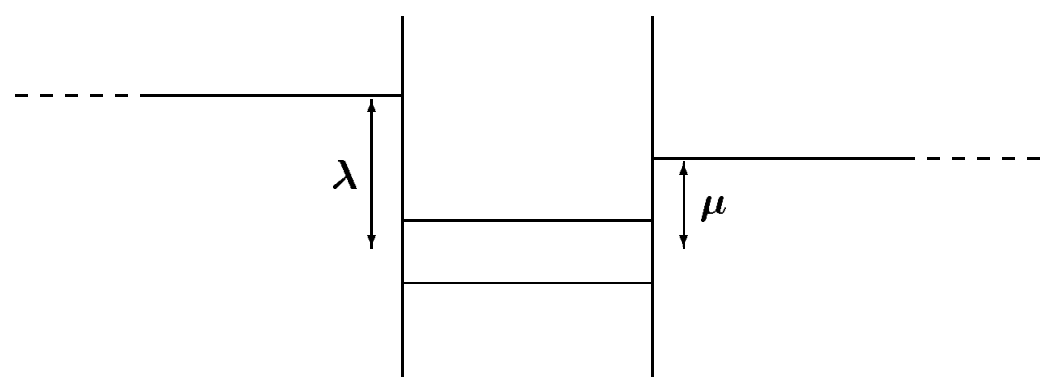

and to quantum field theories with hypermultiplets of masses $\boldsymbol{\lambda}$ and $\boldsymbol{\mu}$. The Dancer space $M(\boldsymbol{\lambda})$ corresponds to



and to quantum field theories with hypermultiplets of mass $\boldsymbol{\lambda}$. These correspondences are described generally in [21]. The reinterpretation of $M(\boldsymbol{\lambda})$ as a fixed monopole moduli space gives an explanation, in the spirit of [21], of the appearance of $M(\boldsymbol{\lambda})$ in [22]. 


\section{ACKNOWLEDGMENTS}

I would like to thank Nick Manton and Paulina Rychenkova for useful discussions and for helpful comments on earlier versions of this paper. I thank the EPSRC and the British Council for financial assistance.

\section{APPENDIX: A NOTE ON ([1],2,[1]) MONOPOLES}

The moduli space of $([1], 2,[1])$ monopoles was used in Sec. V to prove the nonsingularity of $N(\boldsymbol{\lambda}, \boldsymbol{\mu})$. The discussion in Sec. V would also be useful in studying ([1],2,[1]) monopoles per se. All ([1],2, [1]) monopoles are $D_{2}$ symmetric about some axes. The monopole can be orientated by imposing $D_{2}$ symmetry about particular axes. By imposing $D_{2}$ symmetry about the Cartesian axes, the monopoles are restricted to a three-dimensional geodesic submanifold of the moduli space, this is called $\mathbb{X}$. The space $N^{3}$ of Nahm data described above is the quotient of the full moduli space by the full $\mathrm{SO}_{3}$ action and since this action is not free, $N^{3}$ is not a manifold. Instead of quotienting the space of Nahm data by $\mathrm{SO}_{3}, D_{2}$ symmetry is imposed on it, giving $\mathbb{X}$. The $D_{2}$ symmetry conditions are identical to Eq. (37) but without the ordering condition (39). Thus, $\mathbb{X}$ is composed of the six copies of $N^{3}$ obtained by permuting the inequality (39). These copies are joined at the planes where two of the $f_{i}$ 's are equal. These data, where two of the $f_{i}$ 's are equal, correspond to axially symmetric monopoles. The planes intersect on the lines of spherical symmetry. An example of a line of spherical symmetry is

$$
f_{1}(t)=f_{2}(t)=f_{3}(t)=-\frac{1}{t+\tau}
$$

where $\tau>2$.

There are exceptional lines in $\mathbb{X}$ given by letting $k=1$ and taking $\tau$ to infinity. These lines are notable in the context of Sec. $\mathrm{V}$ as the fixed points of the $\mathrm{U}_{1} \times \mathrm{U}_{1}$ action. These are the lines where one $f_{i}$ is constant and the other two are zero. They meet at the point where all three $f_{i}$ are zero. These lines correspond to the exceptional $([1], 2,[1])$ monopoles produced by embedding two $\mathrm{SU}_{2}$ 1-monopoles.

In their paper [23], Dancer and Leese studied the head on collision of $(2,[1])$ monopoles. These collisions are described by geodesics on a two-dimensional manifold that they call $Y$. $\mathbb{X}$ is the analog of $Y$ for ([1], 2, [1]) monopoles. The boundaries of $\mathbb{X}$ occur when $(D, \tau)$ attain the bounds imposed by analyticity. When $(D, \tau)$ attain these bounds, the Nahm data has a pole at one or the other end. This means these boundaries are actually copies of the space $Y$. In fact, the whole of $\mathbb{X}$ has eight copies of $Y$ at its boundaries.

We can picture $\boldsymbol{X}$. Take the $\mathbf{R}^{3}$ Cartesian axes and thicken them. Divide the surfaces of these thickened axes by tracing their intersections with the $x y, y z$ and $z x$ planes. The eight surface elements bounded by these lines are the eight copies of $Y$. The interior of the thickened axes is $X$. The Cartesian axes themselves are the lines of embedded monopoles. The origin is the spherical embedded monopole. The intersections of the six planes $x= \pm y, y= \pm z$ and $z= \pm x$ with $\mathbb{X}$ are the planes of axially symmetric monopoles. The lines $x= \pm y= \pm z$ are the lines of spherically symmetric monopoles. This picture of $\mathbb{X}$ is not metrically correct.

[1] M.F. Atiyah and N.J. Hitchin, 'The geometry and dynamics of magnetic monopoles', (Princeton University Press, Princeton, 1988).

[2] S.A. Connell, 'The dynamics of the $S U(3)$ charge $(1,1)$ magnetic monopoles', ftp://maths.adelaide.edu.au/pure/mmurray/oneone.tex (1994) (unpublished).

[3] J.P. Gauntlett and D.A. Lowe, Nucl. Phys. B472, 194 (1996).

[4] K. Lee, E.J. Weinberg and P. Yi, Phys. Lett. B 376, 97 (1996).

[5] G.W. Gibbons and C. Pope, Commun. Math. Phys. 66, 267 (1979).

[6] A.S. Dancer, Commun. Math. Phys. 158, 545 (1993).

[7] A.S. Dancer, Q. J. Math. 45, 463 (1994).

[8] N.J. Hitchin, A. Karlhede, U. Lindstrom and M. Roček, Commun. Math. Phys. 108, 535 (1987).

[9] W. Nahm, 'The construction of all self-dual multi-monopoles by the ADHM method', in Monopoles in quantum field theory, edited by N.S. Craigie, P. Goddard and W. Nahm, (World Scientific, Singapore, 1982).

[10] N.J. Hitchin, Commun. Math. Phys. 89, 145 (1983). 
[11] H. Nakajima, 'Monopoles and Nahm's equations', Sanda 1990, proceedings, Einstein metrics and Yang-Mills connections edited by T. Mabuchi and S. Mukai (Marcel Dekker, New York, 1993).

[12] J. Hurtubise and M.K. Murray, Commun. Math. Phys. 122, 35 (1989).

[13] E.J. Weinberg, Nucl. Phys. B167, 500 (1980).

[14] R. Bielawski, Nonlinearity, 9, 1463 (1996).

[15] C.J. Houghton and P.M. Sutcliffe, Nonlinearity 9, 1609 (1996).

[16] N.S. Manton, Phys. Lett. B 154, 397 (1985) (E) 157, 475 (1985).

[17] G.W. Gibbons and N.S. Manton, Phys. Lett. B 356, 32 (1995).

[18] K. Lee, E.J. Weinberg and P. Yi, Phys. Rev. D 45, 1633 (1996).

[19] S.W. Hawking, Phys. Lett. A 60, 81 (1977).

[20] M. K. Murray, 'A note on the $(1,1, \ldots, 1)$ monopole metric', hep-th/9605054.

[21] A. Hanany and E. Witten, Nucl. Phys. B492 152 (1997).

[22] N. Seiberg and E. Witten, 'Gauge dynamics and compactification to three-dimensions', hep-th/9607163.

[23] A.S. Dancer and R.A. Leese, Proc. R. Soc. London Ser. A 440, 421 (1993). 